\section{In-vivo characterization of optical properties of pigmented skin lesions including melanoma using oblique incidence diffuse reflectance spectrometry}

\author{
Alejandro Garcia-Uribe, ${ }^{\mathrm{a}, \mathrm{b}}$ Elizabeth B. Smith, ${ }^{\mathrm{c} J u n}$ Zou, \\ Madeleine Duvic, ${ }^{d}$ Victor Prieto, ${ }^{d}$ and Lihong V. Wang ${ }^{a}$ \\ a Washington University in St. Louis, Department of Biomedical \\ Engineering, Optical Imaging Laboratory, St. Louis, Missouri 63130 \\ bTexas A\&M University, Department of Electrical and Computer \\ Engineering, College Station, Texas 77843 \\ 'Texas A\&M University, Department of Biomedical Engineering, \\ College Station, Texas 77843 \\ dThe University of Texas M. D. Anderson Cancer Center, Department \\ of Dermatology, Houston, Texas 77030
}

Abstract. In this letter, we report the first use of oblique incidence diffuse reflectance spectrometry to conduct in-vivo measurements of optical properties of three different types of pigmented skin lesions, including melanoma, dysplastic, and common nevi. Both absorption and reduced scattering coefficient spectra were estimated from the spatially resolved diffuse reflectance within the wavelength range of 455-765 nm for 144 pigmented skin lesions including 16 melanomas. The absorption and reduced scattering spectra were found to change with the malignancy of the skin lesions, which were generally higher for the malignant cases than the benign ones. Based on the measurement results, the physiological origin leading to the change of the absorption and scattering properties is also discussed. (C) 2011 Society of Photo-Optical Instrumentation Engineers (SPIE). [DOI: 10.1117/1.3536509]

Keywords: absorption coefficient; reduced scattering coefficient; diffuse reflectance; and melanoma.

Paper 10556LR received Oct. 12, 2010; revised manuscript received Dec. 16, 2010; accepted for publication Dec. 16, 2010; published online Feb. 17, 2011.

Melanoma is the most malignant type of skin cancer (the most common form of human cancers) and also the leading cause of death from skin diseases. ${ }^{1}$ Early detection and treatment of melanoma can significantly improve patient outcomes. Unfortunately, melanoma is difficult to diagnose by its appearance as it can closely resemble some other pigmented skin lesions, such as common nevi, dysplastic nevi, or seborrheic keratoses. As a result, an invasive biopsy procedure is generally required for accurate diagnosis. Recent studies suggest that the optical properties (e.g., absorption and scattering) of various skin lesions (pigmented or nonpigmented) would change with their different development stages ${ }^{2-5}$ which could be utilized to de-

Address all correspondence to: L. V. Wang, Washington University in St. Louis, Department of Biomedical Engineering, Optical Imaging Laboratory, St. Louis, MO 63130 E-mail: Ihwang@biomed.wustl.edu velop new noninvasive approaches for skin cancer detection. However, the optical properties of melanoma and other pigmented skin lesions have not been well studied or compared up to this date. In this letter, we report the first experimental study of the in-vivo optical absorption and scattering properties of 144 pigmented skin lesions, including 16 melanomas, 98 dysplastic nevi, and 30 common nevi. By using the oblique incidence diffuse reflectance spectroscopy (OIDRS), the spatiospectral diffuse reflectance (within the visible spectrum) of the skin lesions were measured in vivo. The optical absorption and scattering spectra of the skin lesions were then estimated from the measured diffuse reflectance data. Both the absorption and scattering spectra of different types of skin lesions are compared and their pathological implication is also discussed.

The data acquisition was conducted at The University of Texas M.D. Anderson Cancer Center (Houston, TX). The diffuse reflectance spectra were collected from 144 pigmented skin lesions and adjacent healthy skin (for referencing). After the measurement was completed, the skin lesion was biopsied and submitted for histopathological diagnosis. To facilitate the invivo characterization of the pigmented skin lesions, we have developed a unique OIDRS measurement system (Fig. 1). The OIDRS system consists of a white light source, a fiber optic sensor probe, and a data acquisition interface. ${ }^{6}$ The white light source (halogen lamp) provides a full visible wavelength spectrum $(455-765 \mathrm{~nm})$ for the OIDRS measurement. The fiber optic sensor probe consists of one or more source fiber for a $45 \mathrm{deg}$ oblique incidence and two linear array of 10 collection fibers to collect the spatial distribution of diffuse reflectance for the estimation of the absorption and scattering parameters of the skin lesions. ${ }^{6}$ The sensor probe (with an active sensing area of $2 \times 2 \mathrm{~mm}^{2}$ ) was fabricated using advanced microfabrication technology to ensure high precision and performance for the OIDRS measurements even on the smallest skin lesions. The data acquisition interface consists of an imaging spectrograph to resolve the diffuse reflectance spectra (Fig. 2), which is captured by a CCD camera and stored in a personal computer (PC) for subsequent analysis. Before the actual OIDRS measurement was conducted, the entire measurement system was calibrated with optical phantom solutions. ${ }^{7}$

The estimation of the absorption and scattering parameters of the skin lesions from the measured diffuse reflectance was conducted based on a combinative approach based on both the diffusion theory and the scalable Monte Carlo simulation. ${ }^{8}$ In the fiber optic sensor probe, the collection fibers array (detector) is placed in a close position ( $\sim 1 \mathrm{~mm}$ away) from the incidence fiber (light source) to characterize the smallest skin lesions included in this study (limited to $2 \mathrm{~mm}$ in diam). Since the optical transport mean free path $\left(L_{t}^{\prime}\right)$ is a function of the wavelength of the incident light, the location of the detectors may fall either within or outside the range of $L_{t}{ }^{\prime}$ at different wavelengths within the wide spectrum $(455-765 \mathrm{~nm})$ used in the OIDRS measurement. At certain wavelengths, when the location of the detectors falls outside the range of $L_{t}{ }^{\prime}$, the absorption and scattering optical properties of the skin lesion can be directly calculated from the diffuse reflectance using a straightforward diffusion-theory based analytical model. ${ }^{9}$ However, this model would fail at other wavelengths when the detector location falls within $L_{t}{ }^{\prime}$. In this

1083-3668/2011/16(2)/020501/3/\$25.00 (C) 2011 SPIE 


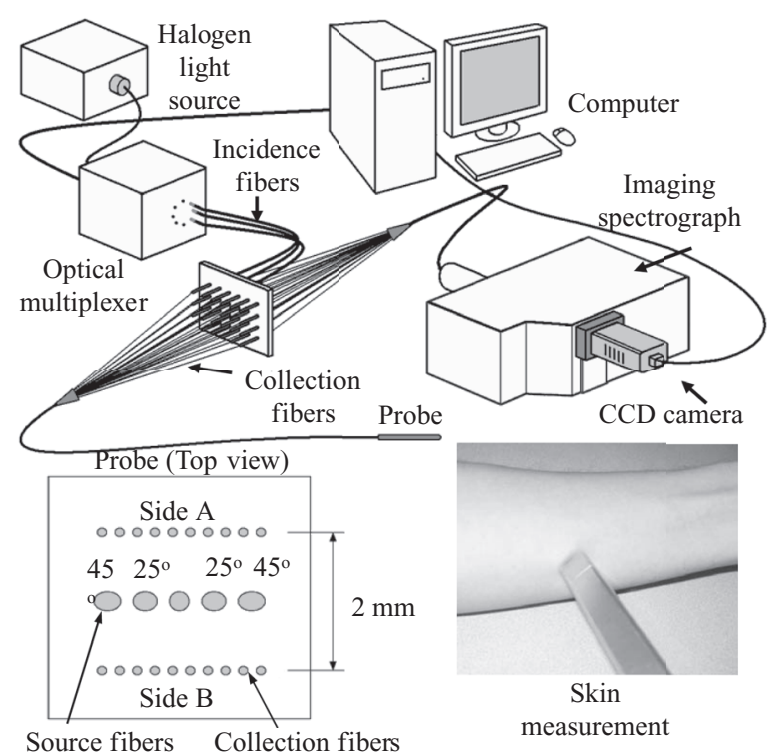

Fig. 1 Oblique incidence diffuse reflectance spectroscopy (OIDRS) system setup.

case, the Monte Carlo simulation can be conducted to deduce the absorption and scattering optical properties of the skin lesions in an inverse problem by calculating and matching the simulated diffuse reflectance results with the actual measurements. However, the Monte Carlo simulation is very inefficient and slow when used in such iterative manner. For this reason, we used the scalable Monte Carlo method. ${ }^{8}$ First, a initial single reference Monte Carlo simulation was conducted with a fixed anisotropy factor $g$. The precalculated time-resolved diffuse reflectance $R(x, y, t)$ was computed for reference absorption and scattering parameters $\left(\mu_{a r}\right.$ and $\left.\mu_{s r}\right)$ and saved in a computer file. The timeresolved diffuse reflectance $R(x, y, t)$ for any new optical parameters, $\mu_{a}$ and $\mu_{s}$, is calculated based on the following relationship

$$
\begin{aligned}
R(x, y, t)= & \left(\frac{\mu_{s}}{\mu_{s r}}\right)^{3} R_{y}\left(x \frac{\mu_{s}}{\mu_{s r}}, y \frac{\mu_{s}}{\mu_{s r}}, t \frac{\mu_{s}}{\mu_{s r}}\right) \\
& \times \exp \left[-\left(\mu_{a}-\mu_{a r}\right) \frac{\mu_{s}}{\mu_{s r}} c t\right] .
\end{aligned}
$$

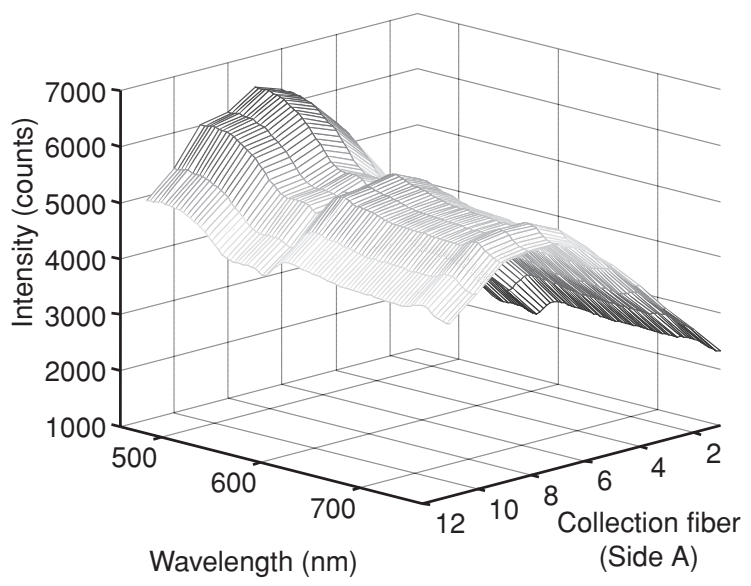

Fig. 2 Sample spatially-resolved diffuse reflectance collected in-vivo form human skin.
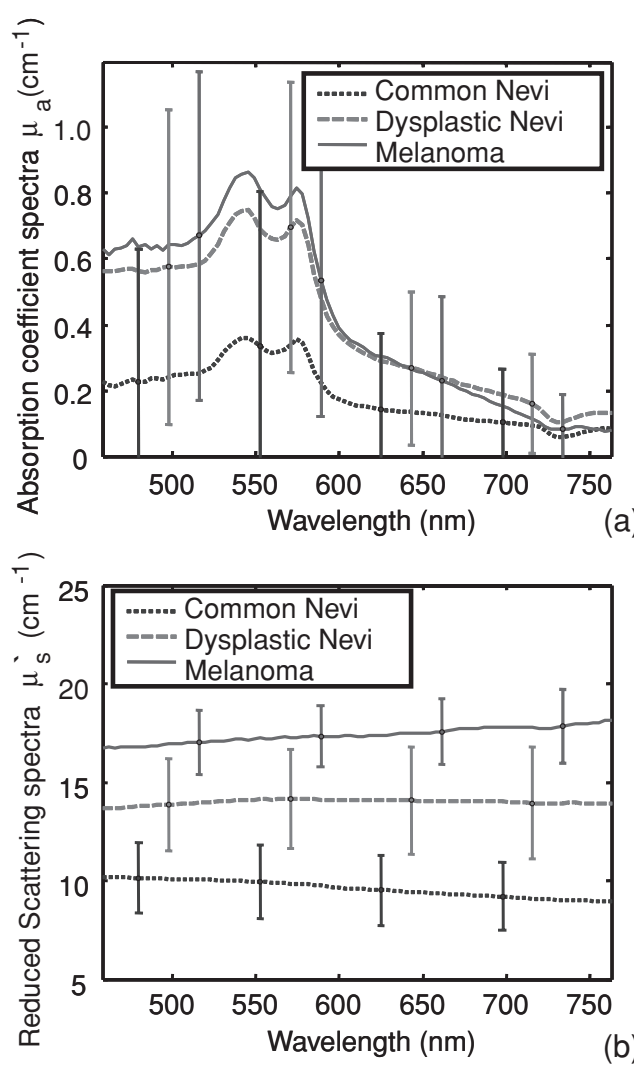

Fig. 3 (a) Average absorption coefficient spectra and average reduced scattering coefficient spectra and (b) for common nevi, dysplastic nevi, and melanoma. The error bars represent standard deviations.

When distance $(y)$ between any collection fiber and the source fiber is constant, $R(x, t)$ is obtained by first scaling in the $y$ direction. The corresponding steady-state diffuse reflectance is calculated by

$$
R(x)=\int_{0}^{\infty} R(x, t) d t .
$$

The reduced scattering and absorption coefficients spectra were estimated for each skin lesion. The average value and standard deviation of the absorption and reduced scattering coefficients for each of the three groups (benign common nevi, dysplastic nevi, and melanoma) are plotted in Figs. 3(a) and 3(b).

As shown in Fig. 3(a), the absorption coefficient spectra for the melanoma and dysplastic cases are similar, which otherwise are generally higher than those for the benign ones. In human skin, the dominant optical absorbers in the visible spectrum range are hemoglobin and melanin. From the absorption coefficient spectra, the concentration of hemoglobin and its oxygen saturation $\left(\mathrm{StO}_{2}\right)$ can be estimated. ${ }^{10}$ The absorption coefficient spectra $\mu_{\mathrm{a}}(\lambda)$ is related to the known extinction coefficients of oxy-hemoglobin $\left(\varepsilon_{\text {mel }}\right)$, deoxy-hemoglobin $\left(\varepsilon_{\text {oxy }}\right)$, melanin $\left(\varepsilon_{\text {oxy }}\right)$ and other absorbers by

$$
\begin{aligned}
\mu_{a}(\lambda)= & \varepsilon_{\text {oxy }}(\lambda) C_{\text {oxy }}+\varepsilon_{\text {deoxy }}(\lambda) C_{\text {deoxy }} \\
& +\varepsilon_{\text {mel }}(\lambda) C_{m e l}+\mu_{a b g},
\end{aligned}
$$

where $C_{\text {oxy }}, C_{\text {deoxy }}$, and $C_{\text {mel }}$ are the concentrations (mMoles) of oxy-hemoglobin, deoxy-hemoglobin, and melanin, and $\mu_{a b g}$ 
Table 1 Differential concentration of hemoglobin and oxygen saturation.

\begin{tabular}{lrr} 
& \multicolumn{1}{c}{$\Delta C_{h b}(\mu \mathrm{M})$} & \multicolumn{1}{c}{$\Delta \mathrm{SO}_{2}(\%)$} \\
\hline $\mathrm{CN}$ & $-1.21 \pm 0.93$ & $-3.5 \pm 2.2$ \\
$\mathrm{DN}$ & $1.17 \pm 0.35$ & $-6.0 \pm 2.6$ \\
$\mathrm{M}$ & $1.34 \pm 0.27$ & $-16 \pm 2.5$ \\
\hline
\end{tabular}

is the absorption caused by other local tissue components and $\lambda$ is the wavelength. The total concentration of hemoglobin $\left(C_{h b}\right)$ is given by

$$
C_{h b}=C_{\text {oxy }}+C_{\text {deoxy }},
$$

and the oxygen saturation is determined by

$$
\text { St } \mathrm{O}_{2}=C_{\text {oxy }} / C_{h b} \text {. }
$$

Because the optical absorption of the human skin could vary significantly between individuals with different race, age, and sun exposure, etc, the differential concentration of hemoglobin and oxygen saturation (i.e., the difference between those of a lesion site and those of its surrounding healthy skin region) thus serves as a more accurate indicator of the tissue malignance. As listed in Table 1, the differential concentration of hemoglobin and oxygen saturation for the melanoma cases is significantly lower than for dysplastic and common nevi cases, which is a clear indication of hypoxia. Lower oxygen saturation exists in the melanoma cases, which could possibly be related to such factors as metabolic abnormalities or abnormal blood supply. ${ }^{11}$

As shown in Fig. 3(b), the reduced scattering coefficient generally increases with the degree of dysplasia or malignancy of the skin lesions within the entire visible spectrum. From the reduced scattering coefficient spectra, the size distributions of the tissue scatterers are estimated using the inverse Mie theory, ${ }^{12}$ while assuming that size distribution of the scatterers is Gaussian $N\left(\mu, \sigma^{2}\right)$ with a mean of $\mu$ and a standard deviation of $\sigma$ (Table 2). It is clearly shown that the melanomas have an average scatterer size significantly larger than those of the dysplastic and benign ones. This result conforms to those obser-

Table 2 Estimated size distribution, $N\left(\mu, \sigma^{2}\right)$ where $\mu$ is the mean and $\sigma$ is the standard deviation.

\begin{tabular}{lcc}
\hline & Mean $\mu(\mu \mathrm{m})$ & Standard deviation $\sigma(\mu \mathrm{m})$ \\
\hline $\mathrm{CN}$ & $6.23 \pm 1.79$ & $1.56 \pm 0.82$ \\
$\mathrm{DN}$ & $8.24 \pm 2.62$ & $2.59 \pm 0.96$ \\
$M$ & $11.01 \pm 3.71$ & $3.35 \pm 1.41$ \\
\hline
\end{tabular}

vations made in previous studies on skin lesions. Mitochondria, other cytoplasmic organelles and cell nuclei, are believed to be the major light scatterers in the skin tissue. ${ }^{13,14}$ The atypical nuclei with larger size existing in the malignant lesions would be a main factor for the significant increase in light scattering.

\section{Acknowledgments}

The authors thank Dr. Mays, Dr. Hymens, and Dr. Mansfield and the staff from the Melanoma and Skin Center at the University of Texas M. D. Anderson Cancer Center for their help during the data collection. This project is sponsored by the National Institute of Health through Grant No. R01 CA106728.

\section{References}

1. American Cancer Society, Cancer Facts \& Figures 2009, http://www.cancer.org.

2. J. R. Mourant, A. H. Hielscher, A. A. Eick, T. M. Johnson, and J. P. Freyer, "Evidence of intrinsic differences in the light scattering properties of tumorigenic and nontumorigenic cells," Cancer 84, 366-374 (1998).

3. Y. N. Mirabal, S. K Chang, E. N. Atkinson, A. Malpica, M. Follen, and R. Richards-Kortum, "Reflectance spectroscopy for in vivo detection of cervical precancer," J. Biomed. Opt. 7, 587-594 (2002).

4. A. Garcia-Uribe, N. Kehtarnavaz, G. Marquez, V. Prieto, M. Duvic, and L. V. Wang. "Skin cancer detection by spectroscopic oblique-incidence reflectometry: classification and physiological origins," Appl. Opt. 43, 2643-2650 (2004).

5. E. Salomatina, B. Jiang, J. Novak, and A. N. Yaroslavsky, "Optical properties of normal and cancerous human skin in the visible and nearinfrared spectral," J. Biomed. Opt. 11(6), 064026 (2006).

6. A. Garcia-Uribe, K. C. Balareddy, J. Zou, and L. V. Wang, "Micromachined fiber optical sensor for in-vivo measurement of optical properties of human skin," IEEE Sensors Journal 8(10), 1698-1703 (2008).

7. G. Marquez and L.-H. Wang, "White light oblique incidence reflectometer for measuring absorption and reduced scattering spectra of tissue-like turbid media," Opt. Express 1, 454-460 (1997).

8. A. Kienle and M. S. Patterson, "Determination of the optical properties of turbid media from a single Monte-Carlo simulation," Phys. Med. Biol. 41, 2221-2227 (1996).

9. L.-H. Wang and S. L. Jacques, "Analysis of diffusion theory and similarity relations," in Photon Migration and Imaging in Random Media and Tissues, B. Chance, R. R. Alfano, Eds., Proc. SPIE 1888, 107-116 (1993).

10. H. Liu, D. A. Boas, Y. Zhang, A. G. Yodh, and B. Chance, "Determination of optical properties and blood oxygenation in tissue using continuous NIR light," Phys. Med. Biol. 40, 1983-1993 (1995)

11. R. S. Cotran, V. Kumar, and T. Collins, Robbins Pathologic Basis of Disease, 6th ed. 1-498, pp. 1170-1268, W. B. Saunders Company, Philadelphia, PA (1999).

12. H. Jiang, G. Marquez, and L.-H. Wang, "Particle sizing in concentrated suspensions by use of steady-state, continuous-wave photon migration techniques," Opt. Lett. 23(5), 394-396, (1998).

13. J. R. Mourant, J. P. Freyer, A. H. Hielscher, A. A. Eick, D. Shen, and T. M. Johnson, "Mechanisms of light scattering from biological cells relevant to noninvasive optical-tissue diagnostics," Appl. Opt. 37, 3586-3593 (1998).

14. J. R. Mourant, M. Canpolat, C. Brocker, O. Esponda-Ramos, T. M. Johnson, A. Matanock, K. Stetter, and J. P. Freyer, "Light scattering from cells: the contribution of the nucleus and the effects of proliferative status," J. Biomed. Opt. 5, 131-137 (2000). 